\title{
Should multisensory temporal acuity be viewed through the window of perceived simultaneity?
}

Kielan Yarrow ${ }^{1}$ and Warrick Roseboom ${ }^{2,3}$

1. City, University of London

2. Sackler Center for Consciousness Science, University of Sussex

3. Department of Informatics, University of Sussex

Temporal coincidence provides a powerful cue that events originating from different sensory modalities, such as audition and vision, have a common cause [1]. Multisensory integration is often found across a range of sub-second physical asynchronies, supporting the existence of a temporal binding window. In recent years, it has become de rigueur to measure temporal binding windows using the simultaneity judgement (SJ) task, and interpret differences between groups as indicative of differences in the temporal sensitivity of integration processes [2-5]. Recently, it was reported that a multisensory deficit (indicated by a widened range of perceived simultaneity) can be observed for audiovisual (but not visuotactile) processing following early visual deprivation [6]. This report is likely of considerable interest to the multisensory community. However, we believe that like similar work [7] it does not include sufficiently explicit caveats about the processes that generate the window of simultaneity, potentially misrepresenting the underlying cause(s) of differences between groups. Here, we advocate more explicit recognition of the fundamentally subjective nature of the 'window' derived from SJs. We argue that it has poor face validity to measure the conceptually distinct temporal binding window, and demonstrate that its extent is dependent on how the observer decides to interpret the word "simultaneous."

The SJ task (Figure 1 for illustrative data) is conceptually akin to the classic detection task, where observers decide whether a weak signal is present or not. In this task, it is tempting to believe that signals become liminal when exceeding some minimal value. Signals below this hard threshold produce the categorical internal state - "I saw nothing". Hence the threshold seems to represent sensitivity.

However, an alternative idea, prominent since the middle twentieth century, is that internal states are continuous, but decision boundaries are applied to generate categorical responses. This debate spawned signal detection theory [8], in which the tendency to declare a stimulus as present, depends upon placement of a decision-level criterion (c), distinguishable from perceptual sensitivity (limited by internal noise) $-d^{\prime}$.

From this perspective, the perceived extent of multisensory asynchrony is also a continuous internal variable (ranging from "sound clearly first" to "light clearly first" in the audiovisual context). The 
range over which an observer judges two stimuli simultaneous depends on the positioning of two decision criteria, below and above exact perceptual synchrony. Sensations falling within this region are categorised as simultaneous, but their underlying perceptual representations remain continuous.

Experience of simultaneity may of course be truly discrete. When stimuli are (intrapsychically) close enough, perception may become categorically "synchronous" without further nuance. We speculate that this account is popular among multisensory researchers - this would explain why the validity of the SJ's window of simultaneity as a measure of the conceptually distinct temporal binding window receives little scrutiny. At face value, the range of values over which I declare two signals to be simultaneous has little claim to measure the range of values at which my brain binds them in order to generate, say, a reaction-time advantage.

We don't know whether hard thresholds exist defining a truly categorical perception of synchrony. However, as illustrated in Figure 1, it is straightforward to demonstrate that the criteria applied to the SJ task when participants first walk into the laboratory are not hard thresholds.

Our participants (supplementary methods) made audiovisual synchrony judgements under standard instructions - respond synchronous if you perceive synchrony (Figure $1 \mathrm{~A} \& \mathrm{D}$ ). Then, a manipulation encouraging the use of tighter decision criteria was introduced: Participants were simply told to be conservative.

The window of simultaneity shrank (Figure 1 B \& E). This was confirmed by fitting data with a model of the SJ task $[9,10]$ and testing how model parameters varied. Parameters representing low and high decision criteria changed significantly (both $\mathrm{t}_{[17]}>4.7, \mathrm{p}<0.001$ ), narrowing the window of simultaneity. Two other model parameters (measuring internal noise) did not change (both $p>$ $0.18)$.

It seems implausible that our instruction would change hard thresholds for perceiving synchrony, or indeed anything akin to sensitivity, except perhaps via training effects. We ruled out this possibility with a post-test in which the original (lack of) instruction was reinstated. Decision criteria rebounded as expected (both $t_{[17]}>3.5, p<0.003$; Figure $\left.1 F\right)$. 
- Data -..... Fit - Prediction with hard perceptual thresholds

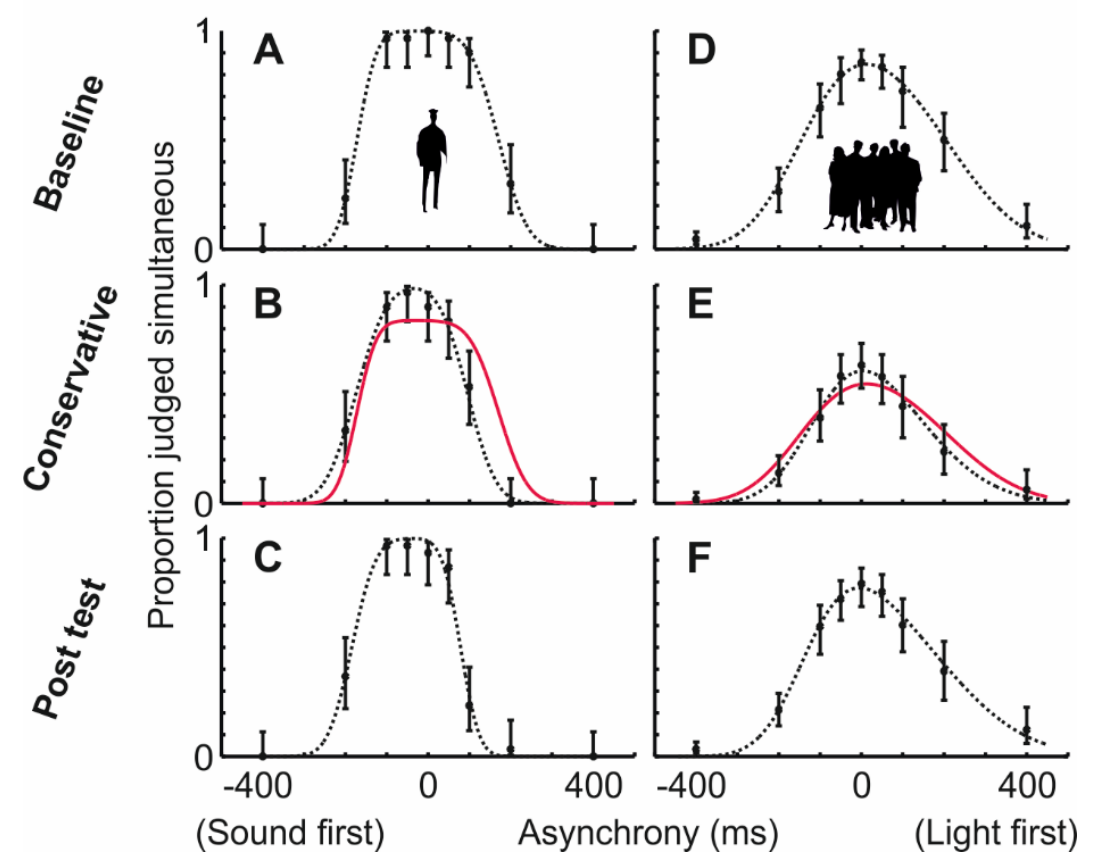

Figure 1. Data, fits and predictions for a simultaneity judgement experiment in which, after a baseline period of data collection, participants were encouraged to "be conservative". A-C. Results for one illustrative participant. Error bars show 95\% binomial confidence intervals. A. At baseline, asynchronies are typically judged synchronous out to around $+/-100 \mathrm{~ms}$. B. Following an instruction to be conservative, less synchronous responses are made, but specifically when sound follows light. This contrasts with the predictions of an account in which participants try to reduce their synchronous responses but continue to be limited by hard thresholds that generate categorical perception (shown in red). C. This particular observer retains their conservative approach even when the instruction is removed. D-F. Results for all participants combined. These are presented purely for illustrative purposes - all model fitting and statistical inference is based on fits to individual participants. Error bars show 95\% bootstrap (BCa) confidence intervals. A. Baseline performance. B. On average, data do not show the reduction in the slope of the psychophysical function predicted by a hard threshold account (shown in red). C. At post-test, most participants relax their criteria again, leading to increased reports of synchrony.

How do we know that decision criteria changed rather than merely seeming to change as an artefact of fitting an inappropriate model to data subverted by unnatural instructions? We predicted what would happen if the thresholds obtained at baseline remained hard. With the "be conservative" instruction enforcing a limited ration of "synchronous" responses, observers would sometimes be 
forced to report asynchrony despite perceiving synchrony. The result would be a uniform (proportional) reduction of the predicted psychometric function (Figure $1 \mathrm{~B} \& \mathrm{E}$, red lines).

Although it does not simulate this behaviour, our psychophysical model can fit this prediction fairly successfully. However, it does so by adjusting all four model parameters, representing the position of both criteria/thresholds and the associated sensory/decision noise. We estimated how much the two noise parameters should have increased under a rationing account. The predicted change was substantial (reducing slope at both sides of the psychometric function) but we found no such change in our participants' data, despite having two attempts to detect it (with around 93\% and 97\% power).

Our data show that windows of perceived synchrony are highly malleable based on decision strategies. Like others, Chen and colleagues [6] have demonstrated that these windows differ between clinical groups. This remains an interesting observation, regardless of why they differ. However, we believe that researchers should point out that these changes do not necessarily reflect perceptual limitations, particularly as any measure prone to strategic alteration is also likely prone to subject and experimenter effects in experiments without double blinding. The window of simultaneity casts only a thin light on multisensory temporal binding processes, and should be interpreted with great caution. Although no panacea, changes in measures reflecting sensitivity seem more pertinent when drawing conclusions about the causes underlying perceptual differences in clinical groups. 


\section{References}

1. Holmes, N.P., and Spence, C. (2005). Multisensory integration: space, time and superadditivity. Curr. Biol. 15, R762-R764.

2. Zampini, M., Guest, S., Shore, D.I., and Spence, C. (2005). Audio-visual simultaneity judgments. Percept. Psychophys. 67, 531-544.

3. Foucher, J., Lacambre, M., Pham, B., Giersch, A., and Elliott, M. (2007). Low time resolution in schizophrenia: lengthened windows of simultaneity for visual, auditory and bimodal stimuli. Schizophr. Res. 97, 118-127.

4. Hillock, A.R., Powers, A.R., and Wallace, M.T. (2011). Binding of sights and sounds: age-related changes in multisensory temporal processing. Neuropsychologia 49, 461-467.

5. Scarpina, F., Migliorati, D., Marzullo, P., Mauro, A., Scacchi, M., and Costantini, M. (2016). Altered multisensory temporal integration in obesity. Sci. Rep. 6, 28382.

6. Chen, Y., Lewis, T.L., Shore, D.I., and Maurer, D. (2017). Early Binocular Input Is Critical for Development of Audiovisual but Not Visuotactile Simultaneity Perception. Current Biology 27, 583589.

7. Wallace, M.T., and Stevenson, R.A. (2014). The construct of the multisensory temporal binding window and its dysregulation in developmental disabilities. Neuropsychologia 64, 105-123.

8. Macmillan, N.A., and Creelman, C.D. (2005). Detection Theory: A User's Guide (New York: Lawrence Erlbaum Associates).

9. Yarrow, K., Minaei, S., and Arnold, D.H. (2015). A model-based comparison of three theories of audiovisual temporal recalibration. Cognit. Psychol. 83, 54-76.

10. Ulrich, R. (1987). Threshold models of temporal-order judgments evaluated by a ternary response task. Percept. Psychophys. 42, 224-239. 
Methods

\section{(a) Participants}

Twenty observers, all naïve to experimental purpose, participated. Data from two participants were not included in the final analysis (see (d) below). Written informed consent was acquired from all participants prior to the experiment, which was approved by the University of Sussex ethics committee. Participants received $f 5$ per hour or course credit as compensation for their time.

\section{(b) Apparatus and materials}

Participants sat in a quiet, bright room. Visual stimuli were displayed on either an liyama Vision Master Pro 203 or LaCie Electron 22 Blue II monitor, both with a resolution of 1024 x 768 pixels and refresh rate of $100 \mathrm{~Hz}$. The monitor was positioned at a viewing distance of approximately $57 \mathrm{~cm}$. Audio signals were presented binaurally through Sennheiser HDA 280 PRO headphones. Stimulus generation and presentation was controlled through Psychtoolbox 3 (Brainard, 1977; Pelli, 1997; Kleiner et al, 2007) run in MatLab (Mathworks, USA) on a desktop PC. Participants responded using the computer keyboard.

Visual events were luminance-modulated Gaussian blobs (standard deviation 1.5 degrees of visual angle (dva)). Peak luminance difference from background was Michelson contrast 1 (Michelson, 1927); displayed against a grey (approx. $38 \mathrm{~cd} / \mathrm{m}^{2}$ ) background. A fixation square (white approx. $76 \mathrm{~cd} / \mathrm{m}^{2}$, subtending $0.25 \mathrm{dva}$ ) was presented centrally. The Gaussian blob was centered $3 \mathrm{dva}$ above the fixation square. The visual stimulus was presented for one frame approximating $10 \mathrm{~ms}$ in duration. Auditory signals were a $10 \mathrm{~ms}$ amplitude pulse of $1500 \mathrm{~Hz}$ sine-wave carrier at approximately $55 \mathrm{db}$ SPL.

\section{(c) Design and procedures}

The experiment consisted of six sessions. Each experimental session took approximately seven minutes to complete. In each session, participants were presented with a sequence of 135 audio-visual presentations. Each presentation consisted of visual and auditory events presented offset by one of nine pseudo-randomly selected stimulus-onset-asynchronies (SOAs) $( \pm 400, \pm 200, \pm 100, \pm 50$ and 0 ms; negative values indicate the auditory event appeared first), with each SOA presented 15 times, interspersed according to the method of constant stimuli. Each audio-visual presentation was preceded by a pseudo-random period of 500-1500 ms. Participants were required to provide an unspeeded response as to whether the auditory and visual events had occurred the same time (synchronously; up cursor key) or not (asynchronously; down cursor key). 
For the first two experimental sessions, these were the only instructions given. Before the third and fourth experimental sessions, participants were told: "Be conservative in your responses. Only press the 'synchrony' key if you are certain". No further guidance was given. Following these two sessions, participants completed two further experimental sessions without any limitations on their responses - the same as the first two sessions completed.

\section{(d) Data analysis}

Data were fitted with an established four-parameter model of the SJ task [1-4]. The observer model assumes that each stimulus is accompanied by Gaussian noise that will affect its central arrival latency, and that the two stimuli comprising an AV pair may be delayed by neural processing to different extents (generating a non-zero point of subjective synchrony). The noisy and delayed signals arrive centrally with latency differences $(\Delta t)$ that form a Normal distribution of internal responses for any physical SOA:

(1) $\Delta t \sim N\left(S O A+\mu, \sigma^{2}\right)$

We assume that two decision criteria are used to interpret $\Delta t$, and that they might also contribute (independent) noise to the decision [5]. If the positions of the two decision criteria (C) are considered Gaussian random variables, the resulting psychometric function is the difference of two cumulative Gaussians:

(2) $\operatorname{Pr}($ "Synchronous" | SOA $)=\Phi\left(C_{\text {High }}, S O A, \sigma_{\text {High }}\right)-\Phi\left(C_{\text {Low }}, S O A, \sigma_{\text {Low }}\right)$

In our analyses, we fitted this model to each participant's data (expressed as proportion judged synchronous at each SOA) in each condition. We first asked whether this four-parameter model provided a significantly better fit (based on a deviance improvement, $\chi_{[2]}^{2}<0.05$ ) than a two parameter cumulative Gaussian, which can capture both guessing and cases where the range of stimuli is sufficient to capture the decision boundary on one, but not both, sides of zero. We excluded one participant because their data did not pass this test in one condition.

We next generated predictions for a hard-threshold model in which participants were assumed to maintain their threshold from the pretest but, in the "be conservative" condition, respond synchronous on a random subset of those trials in which they actually perceived synchrony. 
Predictions were based on the proportional reduction in the use of the synchronous response observed across these two conditions. We excluded one further participant at this stage because their adjustment to the instruction to "be conservative" was to significantly increase their use of the synchronous response $\left(\chi^{2}{ }_{[1]}<0.05\right)$, suggesting they had misunderstood. These predictions were fitted with our four-parameter model in order to generate, for each participant, an estimate regarding how parameters should change under this account. Power analyses for a test of the hard threshold account utilized an effect size estimate derived from the mean of these predicted changes across participants, but normalised by the standard deviation of parameter differences observed between fits to the actual data from pre-test and "be conservative" conditions. 


\section{References}

1. Yarrow, K., Jahn, N., Durant, S., and Arnold, D.H. (2011). Shifts of criteria or neural timing? The assumptions underlying timing perception studies. Conscious. Cogn. 20, 1518-1531.

2. Yarrow, K., Sverdrup-Stueland, I., Roseboom, W., and Arnold, D.H. (2013). Sensorimotor Temporal Recalibration Within and Across Limbs.

3. Yarrow, K., Minaei, S., and Arnold, D.H. (2015). A model-based comparison of three theories of audiovisual temporal recalibration. Cognit. Psychol. 83, 54-76.

4. Yarrow, K., Martin, S.E., Di Costa, S., Solomon, J.A., and Arnold, D.H. (2016). A roving dualpresentation simultaneity-judgment task to estimate the point of subjective simultaneity. Frontiers in psychology 7:416.

5. Ulrich, R. (1987). Threshold models of temporal-order judgments evaluated by a ternary response task. Percept. Psychophys. 42, 224-239. 